Research

Open Access

\title{
Melatonin therapy to improve nocturnal sleep in critically ill patients: encouraging results from a small randomised controlled trial
}

\author{
Richard S Bourne ${ }^{1}$, Gary H Mills² and Cosetta Minelli3
}

\begin{abstract}
1Sheffield Teaching Hospitals, Critical Care Department, Northern General Hospital, Herries Road, Sheffield, UK, S5 7AU
2Sheffield Teaching Hospitals, Critical Care Directorate, Royal Hallamshire Hospital, Glossop Road, Sheffield, UK, S10 2JF

${ }^{3}$ Respiratory Epidemiology and Public Health Group, National Heart and Lung Institute, Imperial College London, Emmanuel Kaye Building, Manresa Road, London, UK, SW3 6LR

Corresponding author: Richard S Bourne, richard.bourne@sth.nhs.uk
\end{abstract}

Received: 8 Feb 2008 Revisions requested: 13 Mar 2008 Revisions received: 11 Apr 2008 Accepted: 18 Apr 2008 Published: 18 Apr 2008

Critical Care 2008, 12:R52 (doi:10.1186/cc6871)

This article is online at: http://ccforum.com/content/12/2/R52

(c) 2008 Bourne et al.; licensee BioMed Central Ltd.

This is an open access article distributed under the terms of the Creative Commons Attribution License (http://creativecommons.org/licenses/by/2.0), which permits unrestricted use, distribution, and reproduction in any medium, provided the original work is properly cited.

\begin{abstract}
Introduction Sleep disturbances are common in critically ill patients and when sleep does occur it traverses the day-night periods. The reduction in plasma melatonin levels and loss of circadian rhythm observed in critically ill patients receiving mechanical ventilation may contribute to this irregular sleepwake pattern. We sought to evaluate the effect of exogenous melatonin on nocturnal sleep quantity in these patients and, furthermore, to describe the kinetics of melatonin after oral administration in this patient population, thereby guiding future dosing schedules.

Methods We conducted a randomised double-blind placebocontrolled trial in 24 patients who had undergone a tracheostomy to aid weaning from mechanical ventilation. Oral melatonin $10 \mathrm{mg}$ or placebo was administered at 9 p.m. for four nights. Nocturnal sleep was monitored using the bispectral index (BIS) and was expressed in terms of sleep efficiency index (SEI) and area under the curve (AUC). Secondary endpoints were SEl measured by actigraphy and nurse and patient assessments. Plasma melatonin concentrations were measured in nine patients in the melatonin group on the first night.

nocturnal sleep (SEl difference $=0.12,95 \% \mathrm{Cl}-0.02$ to $0.27 ; P$ $=0.09$ ) and a decrease in BIS AUC indicating 'better' sleep (AUC difference $=-54.23,95 \% \mathrm{Cl}-104.47$ to $-3.98 ; P=0.04$ ). Results from the additional sleep measurement methods were inconclusive. Melatonin appeared to be rapidly absorbed from the oral solution, producing higher plasma concentrations relative to similar doses reported in healthy individuals. Plasma concentrations declined biexponentially, but morning (8 a.m.) plasma levels remained supraphysiological.

Conclusion In our patients, nocturnal sleep quantity was severely compromised and melatonin use was associated with increased nocturnal sleep efficiency. Although these promising findings need to be confirmed by a larger randomised clinical trial, they do suggest a possible future role for melatonin in the routine care of critically ill patients. Our pharmacokinetic analysis suggests that the 10-mg dose used in this study is too high in these patients and may lead to carryover of effects into the next morning. Reduced doses of 1 to $2 \mathrm{mg}$ could be used in future studies.
\end{abstract}

Results Nocturnal sleep time was 2.5 hours in the placebo group (mean $\mathrm{SEI}=0.26,95 \%$ confidence interval $[\mathrm{Cl}] 0.17$ to

Trial registration Current Controlled Trials ISRCTN47578325.

\section{Introduction}

Sleep disturbances are common in critically ill patients, who present a loss of monophasic nocturnal sleep combined with frequent diurnal naps (irregular sleep-wake pattern) [1] as well as a reduction in deeper, more restorative phases such as slow-wave sleep (SWS) and rapid eye movement (REM) sleep [2]. Although the consequences of such prolonged sleep fragmentation are unknown, they may be comparable to the signif-

$\overline{\mathrm{AUC}}=$ area under the curve; $\mathrm{AUC}_{(0-24)}=$ area under the concentration time curve between time 0 and 24 hours; $\mathrm{BIS}=$ bispectral index; $\mathrm{C}_{\max }=$ maximum plasma concentration; CYP1A2 = cytochrome P450 1A2; EEG = electroencephalogram; ICU = intensive care unit; RCSQ = Richards Campbell Sleep Questionnaire; REM = rapid eye movement; SAS = Sedation Agitation Scale; SD = standard deviation; SEI = sleep efficiency index; SWS $=$ slow-wave sleep. 
icant morbidity associated with prolonged sleep deprivation [3]. Patients themselves perceive sleep disturbances to be one of the most stressful components of their intensive care stay [4].

Nocturnal secretion of melatonin synchronises the sleep-wake and dark-light cycles [5], and disruption to the normal timing and amplitude of the circadian rhythm of melatonin secretion is associated with reduced sleep $[6,7]$. Reduction in plasma melatonin levels and lack of circadian rhythm have been shown in critical care patients undergoing mechanical ventilation [811].

Exogenous melatonin has been demonstrated to be safe and effective in the treatment of other circadian rhythm sleep disorders [12]. This study aimed to examine the effect of exogenous melatonin on nocturnal sleep in patients being weaned from mechanical ventilation. The optimum oral dose to use in this population is also unknown and therefore a pharmacokinetic analysis of plasma melatonin concentrations was also undertaken.

\section{Materials and methods}

We conducted a randomised double-blind placebo-controlled trial in patients admitted to an adult general intensive care unit (ICU) with acute respiratory failure requiring mechanical ventilation and tracheostomy to assist weaning. Exclusion criteria were an expected ICU length of stay of less than 5 days, preadmission treatment of sleep disturbances, contraindications to enteral feeding, a history of convulsions, psychiatric or neurological disease, alcohol consumption of greater than or equal to 50 units per week or drug use, sleep apnoea, severe heart failure (New York Heart Association classification III/IV), and low levels of consciousness, defined as values of below 4 on the Sedation Agitation Scale (SAS) [13]. The local ethics committee approved the study protocol and all patients provided written informed consent.

Patients were randomly assigned to melatonin or placebo by the pharmacy, using random assignment in blocks of four. Melatonin $10 \mathrm{mg}$, formulated in an oral liquid, or matching placebo was administered enterally at 9 p.m. for four consecutive nights [14]. Propofol and alfentanil were discontinued at least 30 hours, and morphine and midazolam at least 48 hours, before study entry. No hypnotics were allowed during the study. Haloperidol was allowed in very agitated patients (SAS of greater than or equal to 6). Earplugs and eye masks were made available for use at the patients' discretion, and staff meetings and posters were employed to encourage staff to minimise environmental, nursing, and clinical disturbances during the nocturnal study periods. Environmental disturbances were documented based on a locally derived scale composed of light interruptions, clinical activities, and use of invasive instrumentation (Additional file 1). The nurses also subjectively ranked the noise level each night (Additional file
1). Baseline nocturnal illuminance at the head of each patient bed when all lights were off was recorded using a light meter (Luxmeter PU150; Eagle International, Wembley, UK). Drug records were compiled daily for drugs known to adversely affect sleep [15] or melatonin pharmacokinetics [16].

\section{Sleep measurement}

Nocturnal sleep was evaluated using the bispectral index (BIS) (BIS XP, Quattro sensor; Aspect Medical Systems, Inc., Norwood, MA, USA), a signal-processing technique based on the electroencephalogram (EEG) previously used to evaluate sleep in critical care patients [17]. BIS data were recorded in 5-second intervals and downloaded onto a personal computer. Two outcome measures were used: sleep efficiency index (SEI) and area under the curve (AUC). SEl was defined as the ratio of a patient's total sleep time over the time available for 'nocturnal' sleep (9 hours, from 10 p.m. to 7 a.m., corresponding to nursing staff shift patterns). Sleep was defined as BIS below 80 [18]. AUC was calculated using the trapezoidal rule, which uses trapeziums to approximate the region under a curve and calculate its area. For each night, SEI and AUC values were set to missing if recordings were missing for more than 2 hours. Analyses were limited to nights 3 and 4 since the potential chronohypnotic benefits of melatonin are not immediate and may take 3 days to be realised [19,20]. All four nights were considered in a secondary analysis.

During the study, other sleep measurement methods were used with the main aim of evaluating agreement and comparing feasibility and reliability in the critical care setting. These included actigraphy (Actiwatch; Cambridge Neurotechnology Ltd., Cambridge, UK), nurse assessment (direct nurse observation using hourly epochs), and patient assessment (Richards Campbell Sleep Questionnaire [RCSQ]). Details of the methods and results on measurement agreement are reported elsewhere [21]. Results of these methods for the melatonin effect, expressed in terms of SEl, are reported here as secondary analyses.

\section{Statistical analysis}

Differences between treatment groups in mean values of SEI and $A U C$, averaged over nights 3 and 4 , were analysed using the $t$ test with equal variances. For the secondary analysis, including all four nights, we used a multilevel model, Prais regression, which accounts for the within-patient correlation between measurements on successive nights. Mean and standard deviation (SD) or median and interquartile range were used as appropriate for descriptive statistics. The Pearson correlation was used for test of association. Data were analysed using Stata 9.1 software (StataCorp LP, College Station, TX, USA).

A sample size of 34 patients was calculated based on BIS SEl, assuming $\alpha=0.05$, power $=0.8$, and minimum detectable difference in SEI $=0.20$. Since no data on the SD of BIS SEI in 
critical care patients were available, we used the SD of SEI obtained using polysomnography as a proxy. Polysomnography studies reported SD values from 0.1 to 0.24 [2,22-24] and we used a conservative value of 0.20 .

\section{Pharmacokinetic analysis}

Pharmacokinetic analysis of plasma melatonin concentrations was undertaken in the first nine patients in the melatonin group. Twelve blood samples were collected from each patient at appropriately spaced intervals after the first oral dose. All samples were taken from the arterial line, immediately centrifuged, and stored at $-20^{\circ} \mathrm{C}$ until assay. Plasma melatonin was measured in duplicate using a melatonin direct radioimmunoassay (Immuno Biological Laboratories, Hamburg, Germany). Sample dilution to within the linear range of the assay was undertaken as necessary. The values of intra-assay precision (percentage coefficient of variation) at plasma concentrations of approximately 10 and $150 \mathrm{pg} / \mathrm{mL}$ were $13.6 \%$ and $6.8 \%$, respectively. The interassay coefficient of variation was 24.5\%. Plasma concentrations were corrected for endogenous plasma melatonin concentration by subtracting the 9 p.m. baseline value. Non-compartmental pharmacokinetic analysis was undertaken (PKSolution 2.0; Summit Research Services, Montrose, CO, USA).

\section{Results}

Figure 1 shows patients' inclusion in the study. Due to slow recruitment, we could recruit only 24 patients. There were 4 patients ( 3 in the placebo and 1 in the melatonin group) with missing data for nights 3 and 4 , the reasons being discharged/ re-sedated (4 nights), patient removed sensor (2 nights), signal quality index low (1 night), and patient refused (1 night).

Table 1 shows patients' baseline characteristics in the two treatment groups. An imbalance of known risk factors for sleep disturbances was present due to small sample size, potentially leading to more sleep disturbance in the melatonin group. Such factors included older age [25], delirium [26], and ventilation with pressure support ventilation (because of the possibility of desynchrony) [27]. No differences between the melatonin and control groups were observed with regard to either patient uptake of earplugs or eye masks ( $9 \%$ and $2 \%$ of nights, respectively) or nocturnal environmental disturbances score. The mean (SD) baseline illuminance at the head of each

\section{Figure 1}

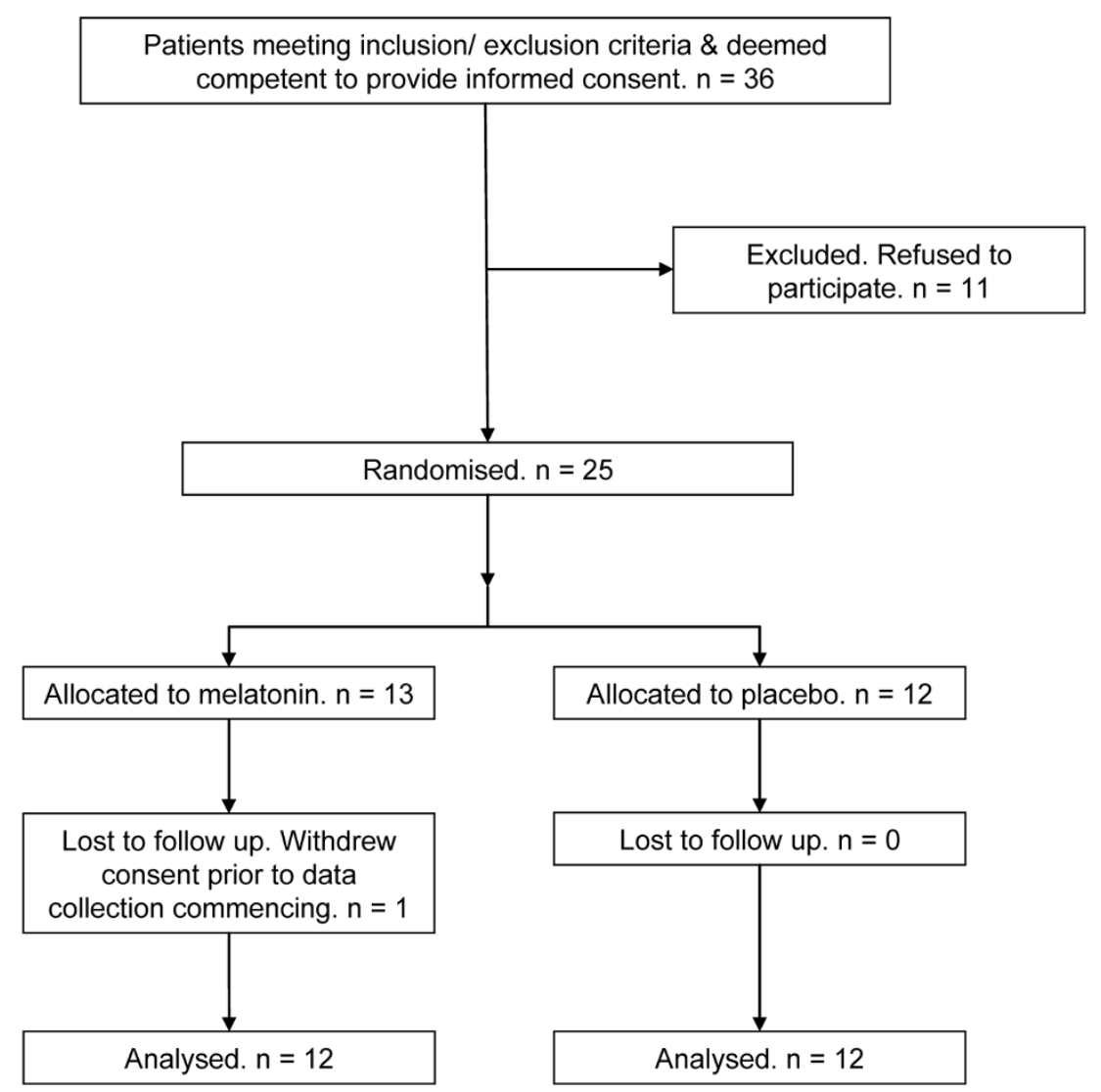

Flowchart of the study, from patient recruitment to analysis. 
Table 1

\begin{tabular}{|c|c|c|}
\hline Characteristic & Placebo $(n=12)$ & Melatonin $(n=12)$ \\
\hline Male, number (percentage) & $7(58.3)$ & 4 (33.3) \\
\hline \multicolumn{3}{|l|}{ Reason for ICU admission, number (percentage) } \\
\hline Severe sepsis & $8(66.7)$ & $10(83.3)$ \\
\hline Postoperative respiratory failure & $2(16.7)$ & $1(8.3)$ \\
\hline Pneumonia & $2(16.7)$ & $1(8.3)$ \\
\hline Age in years, mean (SD) & $58.7(12.5)$ & $69.9(12.0)$ \\
\hline APACHE II score on study entry, mean (SD) & $16.8(3.4)$ & $17.3(3.8)$ \\
\hline Actual body weight in kilograms, median (IQR) & $69.0(57.4 ; 77.5)$ & $65.0(63.5 ; 70.0)$ \\
\hline Ideal body weight in kilograms, mean (SD) & $60.0(6.9)$ & $57.2(6.5)$ \\
\hline Body mass index, mean (SD) & $24.6(4.7)$ & $25.0(3.1)$ \\
\hline Patients' usual sleep quantity in hoursa, mean (SD) & $6.5(1.57)$ & $6.2(2.07)$ \\
\hline ICU length of stay prior to study in days, median (IQR) & $16.5(13.0 ; 20.5)$ & $16.5(11.0 ; 19.0)$ \\
\hline Time of ventilation prior to study in days, mean (SD) & $20.0(14.3)$ & $13.6(6.5)$ \\
\hline Sedation (morphine/midazolam) prior to study, number (percentage) & $2(16.7)$ & $2(16.7)$ \\
\hline Time since sedation stopped prior to study in days, mean (SD) & $6.6(2.9)$ & $7.5(4.7)$ \\
\hline Delirium during study period, number (percentage) & $1(8.3)$ & 4 (33.3) \\
\hline \multicolumn{3}{|l|}{ Ventilation mode on nights 3 and 4 , number (percentage) } \\
\hline BiPAP/CPAP-ASB & $7(70.0)$ & $7(58.3)$ \\
\hline External CPAP/Hi-flow oxygen & $3(30.0)$ & $5(41.7)$ \\
\hline
\end{tabular}

aUsual sleep time at home as reported by the patient. APACHE II, Acute Physiological and Chronic Health Evaluation II; BiPAP, biphasic positive airway pressure; CPAP, continuous positive airway pressure; CPAP-ASB, continuous positive airway pressure with assisted spontaneous breathing; ICU, intensive care unit; IQR, interquartile range; SD, standard deviation.

bed when all lights were turned off was 9.6 (2.6) lux.

There was no disparity between the groups in their exposure to the number of potentially sleep-disruptive medications. In patients who received morphine and midazolam, sufficient time elapsed between discontinuation of sedation and study enrolment to limit the potential distortion of results due to accumulation of these agents. None of the patients received haloperidol on nights 3 or 4 . Nocturnal sleep time did not seem to correlate with patients' severity of illness, as measured by APACHE II (Acute Physiological and Chronic Health Evaluation II) daily score, although the wide confidence interval does not allow us to draw definitive conclusions $(r=0.10$; 0.36 to $0.52 ; P=0.68$ ).

Results of the effect of melatonin on primary and secondary sleep measurements are shown in Table 2. Nocturnal sleep time was 2.5 hours in the placebo group and was 1 hour longer in the melatonin group, although the difference was not statistically significant (Table 2). BIS AUC showed a statistically significant $7 \%$ decrease in the melatonin group, with lower AUC meaning 'better' sleep (AUC difference = -54.23; -104.47 to $-3.98 ; P=0.04)$. To account for the imbalance in baseline characteristics, we adjusted the analyses using linear regression. The small sample size limited the number of covariates we could adjust for [28] and we thus created a single variable indicating the overall baseline risk of sleep disturbances. High risk was defined as the presence of any two of the following: age of greater than or equal to 70 years, delirium positive, and ventilation with BiPAP (biphasic positive airway pressure) or CPAP-ASB (continuous positive airway pressure with assisted spontaneous breathing). The results of the adjusted analysis did not vary substantially, apart from an expected loss in precision of the estimates: SEl difference $=0.12(-0.04$ to $0.28 ; P=0.12)$ and AUC difference $=-48.76(-103.06$ to $5.54 ; P=0.07)$. Any evidence of a treatment effect nearly disappeared when considering all four nights: SEl difference $=$ 0.05 ( -0.07 to 0.17$)$ and $A U C$ difference $=-26.62(-70.51$ to 17.28). Results from the additional sleep measurement methods did not support those obtained with BIS and indeed were all inconclusive (Table 2). As regards possible side effects of melatonin, one patient in the melatonin group reported a headache on a single night, which responded to acetaminophen administration. 
Table 2

Effect of melatonin on nocturnal sleep efficiency on nights 3 and 4, using different outcome measures

\begin{tabular}{lcccc}
\hline & \multicolumn{4}{c}{ Bispectral index sleep efficiency index (95\% confidence interval) } \\
\cline { 2 - 5 } Sleep measurement method & Placebo group & Melatonin group & Difference & $P$ value of the difference \\
\hline $\begin{array}{l}\text { Primary analysis } \\
\text { Bispectral index }\end{array}$ & $0.26(0.17$ to 0.36$)$ & $0.39(0.27$ to 0.51$)$ & $0.12(-0.02$ to 0.27$)$ & 0.09 \\
Secondary analysis & & & & 0.84 \\
Actigraphy & $0.75(0.67$ to 0.83$)$ & $0.73(0.53$ to 0.93$)$ & $-0.02(-0.24$ to 0.20$)$ & 0.58 \\
Nurse assessment & $0.51(0.35$ to 0.68$)$ & $0.45(0.26$ to 0.64$)$ & $-0.06(-0.29$ to 0.17$)$ & 0.32 \\
Patient assessment (RCSQ) & $0.50(0.43$ to 0.58$)$ & $0.41(0.24$ to 0.59$)$ & $-0.09(-0.28$ to 0.09$)$ & \\
\hline
\end{tabular}

RCSQ, Richards Campbell Sleep Questionnaire.

The main pharmacokinetic data are summarised in Table 3. Plasma melatonin concentrations declined bi-exponentially (Figure 2). Both maximum plasma concentration $\left(\mathrm{C}_{\max }\right)$ and $\mathrm{AUC}_{(0-24)}$ (area under the concentration time curve between time 0 and 24 hours) had a moderately strong correlation with plasma alanine transaminase concentrations $(r=0.70 ; 0.06$ to $0.93 ; P=0.04$, and $r=0.62 ;-0.07$ to $0.91 ; P=0.07$, respectively). No such association was found with age, gender, weight, creatinine, or bilirubin. No association was found between the pharmacokinetic parameters: $C_{\max }, \mathrm{AUC}_{(0-24)}$ or $\mathrm{C}_{(08)}$ (plasma concentration at 8 a.m.), and mean SEI or BIS AUC measurements of nocturnal sleep.

\section{Discussion}

Our study confirms previous findings $[17,29]$ that nocturnal sleep in patients being weaned from mechanical ventilation is highly compromised, with an average of only 2.5 hours in the placebo group. Melatonin therapy was associated with a 1-

\section{Figure 2}

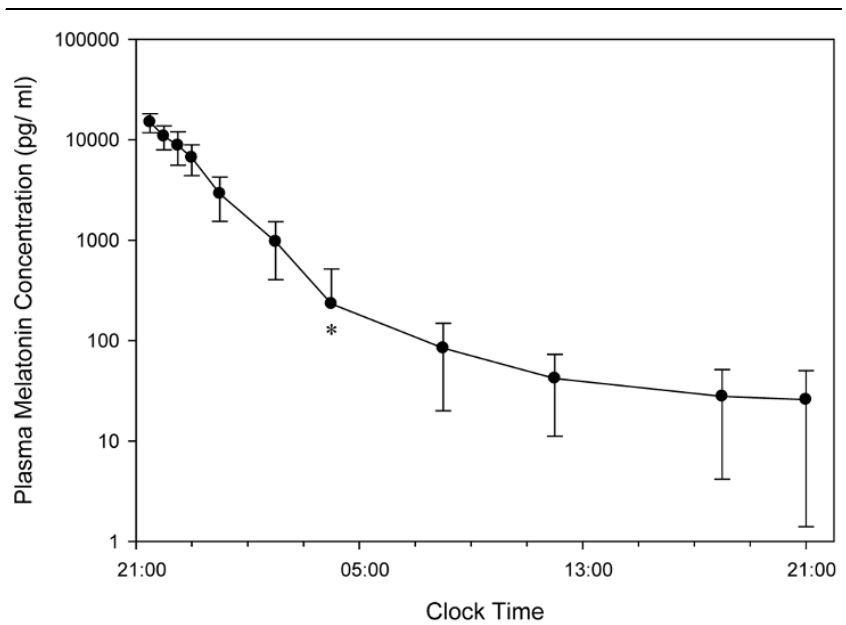

Semi-logarithmic plot of mean melatonin plasma concentration ( \pm standard deviation $[ \pm$ SD]) versus clock time after a $10-\mathrm{mg}$ oral solution dose administered at 9 p.m. in critical care patients. * 4 a.m. data point. Mean concentration value minus SD is a negative number and cannot be represented on a logarithmic scale. hour increase in nocturnal sleep compared with placebo, corresponding to an increase of $47 \%$, although the SEl difference did not reach statistical significance. We found a statistically significant reduction of $7 \%$ in BIS AUC with melatonin administration, suggesting sleep improvement. The use of AUC has some advantages compared with SEl. Apart from providing greater statistical power, BIS AUC provides an indication of both sleep quantity and quality [17], which might be more informative than sleep quantity alone. However, the clinical significance and interpretation of a reduced AUC remain unclear [30].

Two other small trials investigated the effect of melatonin on nocturnal sleep in critically ill patients $[11,31]$, but comparison is limited due to the use of different sleep measurement methods, for which agreement is rather poor [21]. In fact, although polysomnography is the gold standard for quantifying and qualifying sleep, the challenges of the critical care environment have led to the use of a number of alternative methods [21]. The first study was a crossover trial that used actigraphy on eight respiratory patients and showed positive results [31]. Baseline sleep was reported to increase from approximately 3 to 6 hours with melatonin administration, although results of the comparison between melatonin and placebo were not reported. The second study used nurse observation to evaluate 32 tracheostomised patients and showed negative results [11]. Placebo patients slept for about 4 hours, with only 15 minutes more in the melatonin group. As a measure of sleep, actigraphy is not ideal in critically ill patients, being influenced by abnormalities of the neuromuscular system which are common in these patients [21]. As regards nurse observation, intensive observation of sleep (5-minute intervals) is probably necessary to allow differentiation between interventions in critical care studies [32] and even then it suffers from being a subjective measure that may overestimate sleep quantity [33]. Patient assessment has been used in critical care sleep studies on other interventions but its applicability is limited by patients' acute cognitive and perceptual problems [21]. We chose to use BIS as the primary outcome measure since it pro- 
Table 3

Summary of main pharmacokinetic results

\begin{tabular}{cccccc}
\hline $\mathrm{T}_{\max }$, hours & $\mathrm{C}_{\max }, \mathrm{pg} / \mathrm{mL}$ & $\mathrm{AUC}_{(0-24)}, \mathrm{ng}$-hours/L & Overall $\mathrm{t}_{1 / 2}$, hours & Oral clearance $(\mathrm{Cl} / \mathrm{F}), \mathrm{L} / \mathrm{hour}$ & $\mathrm{C}(08), \mathrm{pg} / \mathrm{mL}$ \\
\hline $0.5(0)$ & $14,974(3,200)$ & $29,979(8,205)$ & $1.47(0.28)$ & $351.0(96.7)$ & $84(64)$ \\
\hline
\end{tabular}

Data are presented as mean (standard deviation). $\mathrm{AUC}_{(0-24)}$, area under the concentration time curve between time 0 and 24 hours; $\mathrm{C}_{(08)}$, plasma concentration at 8 a.m.; $\mathrm{Cl} / \mathrm{F}$, clearance/bioavailability (oral dose/area under the zero moment curve); $\mathrm{C}_{\max }$, maximum plasma concentration; $\mathrm{t}_{1 / 2}$, plasma half-life; $\mathrm{T}_{\max }$.

vides an objective measure of sleep which is not adversely affected by the presence of neuromuscular weakness. However, the BIS, similar to other EEG-based techniques, can be adversely affected by conditions such as traumatic brain injury, dementia, or delirium which result in EEG slowing [21]. Although we used BIS XP technology, a degree of susceptibility to increased BIS values as a consequence of electromyogram artefact remains [34]. In our study, the results from actigraphy, nurse observation, and patient assessment, which we used as secondary outcome measures, were all inconclusive. Differences between our BIS SEI results and those of our other measures may be explained somewhat by residual neuromuscular weakness in patients recovering from sepsis (actigraphy), the use of hourly epochs (nurse assessment), and limitations in the patients' ability to complete the RCSQ (patient assessment) [21], all of which may lead to overestimates of sleep quantity and SEl.

Melatonin appears to have a favourable adverse effect profile; headaches, dizziness, nausea, and drowsiness are the most common adverse events reported with short-term melatonin administration [35]. Melatonin treatment appeared to be well tolerated in our patients, with only one patient reporting a single episode of headache.

Melatonin appeared to be rapidly absorbed from the oral solution, and peak concentrations were higher than those reported for comparable doses in healthy individuals [36,37]. After oral dosing, the $C_{\max }$ is affected by the solubility of melatonin in the formulation, alterations in bioavailability, and clearance. Orally administered melatonin is subject to an extensive 'first-pass effect', with bioavailability reported to be approximately $15 \%$ [38], although there is high variability due to factors such as cytochrome P450 1A2 (CYP1A2) activity and co-administration of interacting drugs [39]. The acute inflammatory cascade related to sepsis may adversely affect cytochrome $\mathrm{P} 450$ regulation, including CYP1A2 enzyme activity $[40,41]$, and a prolonged reduction in enzyme function in patients recovering from critical illness may have contributed to the high peak concentrations. Conversely, the high $\mathrm{C}_{\max }$ and $\mathrm{AUC}_{(0-24)}$ could not be accounted for by concurrent use of CYP1A2 inhibitors. Although conventional liver function tests are poor predictors of hepatic drug metabolism, there was a moderate correlation between plasma transaminase levels and measures of exogenous melatonin exposure. Contrary to a report of endogenous plasma levels in cirrhotic patients [42], no such association was found for total bilirubin, although the power of our analysis was limited.

We also found no association between markers of drug exposure and nocturnal sleep quantity. The soporific and entraining effects of melatonin have been shown to reach a plateau at plasma concentrations lower than those described in our patients [43]. Therefore, having plasma concentrations in excess of the dose-dependent range would not be expected to demonstrate further improvements in sleep efficiency. The ideal dosing schedule of melatonin would produce an appropriate rapid peak plasma concentration while maintaining 'physiological' plasma levels over the nocturnal period. Our patients were unable to receive a modified release formulation, being fed via enteral feeding tubes, and hence we used a relatively large immediate-release formulation to ensure continuous nocturnal exposure. As described by others [44], the administered dose resulted in some patients with relatively low clearance having potentially 'nocturnal' plasma levels during the late morning. This may have negated some of the potential chronotherapeutic benefits of melatonin [12]. The presence of supraphysiological levels in the morning will have a phase-delaying effect and thereby negate some of the benefits of the phase-advancing effect of the 9 p.m. administration. However, we did not find an inverse correlation between nocturnal sleep markers and melatonin plasma concentration at 8 a.m. as might therefore be expected. Our pharmacokinetic data suggest that immediate-release doses of 1 to $2 \mathrm{mg}$ administered at 9 a.m. might provide suitable nocturnal plasma melatonin concentrations whilst minimising the risk of daytime overdose.

\section{Limitations of the study and suggestions for future research}

There are a number of obvious limitations in our study which should be reviewed when considering the methodology of future studies. The study was smaller than planned, with only $71 \%$ of the target sample size being reached, mainly due to problems in obtaining consent in the most acutely ill patients. Statistical power was further decreased because of missing data. Both of these problems should be taken into account when designing a study, particularly in deciding on the inclusion criteria and complexity of the study protocol. The small sample size also meant that we had imbalances in baseline characteristics between the groups, although our attempt to 
adjust for important sleep-related factors (age, delirium, and ventilator status) did not materially alter the results.

Our use of alternative sleep measurement techniques to polysomnography also limited the scope of our results. We did not have sleep-stage data and therefore cannot comment on the effect of melatonin on SWS or REM sleep phases. The ultimate aim of sleep interventions in critical care patients is to attempt to consolidate nocturnal sleep and increase both SWS and REM sleep phases. At low doses, melatonin has a sleep-promoting effect without a significant adverse effect on normal sleep architecture [45], a potential advantage over conventional hypnotic agents. Indeed, it could be suggested that the improvements in sleep quantity observed may have been achieved with a conventional hypnotic agent (for example, zopiclone). The significant potential for adverse cognitive effects of these agents, particularly in older patients [46], still makes melatonin (or melatonin agonists such as ramelteon) worth continued investigation.

Ideally, polysomnography should be used as a continuous measure of sleep in further studies. However, such an application presents significant logistical and technical challenges and is associated with specific difficulties, including patient tolerability and sleep-stage interpretation in patients experiencing complex electrophysiological changes [17].

We did not have a useful measure of daytime sleep because our actigraphy data significantly overestimated nocturnal and diurnal sleep quantity [21] and our BIS recording was restricted to the nocturnal period due to patient tolerability. We are therefore unable to comment on the effect of melatonin on daytime sleep. While we are primarily interested in optimising nocturnal sleep with interventions, we should not ignore the potential impact that diurnal sleep periods have on nocturnal sleep efficiency. Approximately half of total sleep time of critical care patients may occur during the diurnal period, with significant inter- and intra-patient variability as to whether sleep deprivation is present over 24 hours [3].

Our environmental score provided only a guide to nocturnal patient disturbances. Noise, light, and patient disturbances have been shown to account for approximately $30 \%$ of nocturnal arousals and awakenings [47]. Although the ambient nocturnal illuminations were at an appropriate level to allow normal melatonin secretion [48], we did not have an accurate measure of light interruptions. The absence of continuous light and noise measurements and lack of quantification of patient disturbances by staff are therefore further potential limitations. Earplugs can improve sleep quality in healthy volunteers exposed to simulated intensive care noise [49]. However, we found that patient willingness to use eye masks and/or earplugs was very low, which limits their routine clinical application. Finally, future studies should consider extending the sleep intervention to a coordinated bright light and exogenous mela- tonin therapy. The sleep-wake process relies on a combination of homeostatic and circadian factors for its optimum function [50], and the full activity of melatonin on the sleep-wake cycle in humans requires the coordination of other time cues such as light [12].

\section{Conclusion}

Although suggesting a possible future role of melatonin in the routine care of critically ill patients, our findings need to be confirmed by a larger, possibly multicentre, randomised controlled trial, ideally using polysomnography as a continuous measure of sleep quantity and quality. A 10-mg nocturnal dose of melatonin is excessive in this patient population and reduced doses of 1 to $2 \mathrm{mg}$ could be used in future chronotherapeutic studies.

\section{Key messages}

- Nocturnal sleep quantity in patients being weaned from mechanical ventilation is highly compromised.

- Melatonin therapy may increase nocturnal sleep quantity, but further investigation using continuous polysomnography is necessary to provide sleep quality information.

- A 10-mg dose of melatonin produces supraphysiological morning plasma levels in critical care patients, possibly negating some of the phase-advancing effects of nocturnal administration.

- Immediate-release doses of 1 to $2 \mathrm{mg}$ administered at 9 p.m. might provide suitable nocturnal plasma melatonin concentrations whilst minimising the risk of daytime overdose.

\section{Competing interests}

The authors declare that they have no competing interests.

\section{Authors' contributions}

RSB conceived the clinical study, enrolled patients, collated the data, and analysed and interpreted the results. GHM participated in the design of the clinical study and the data analysis. CM completed the statistical analysis and assisted with the interpretation of results. All authors contributed to, read, and approved the final manuscript.

\section{Additional files}

The following Additional files are available online:

\section{Additional file 1}

Environmental disturbances log

See http://www.biomedcentral.com/content/ supplementary/cc6871-S1.doc 


\section{Acknowledgements}

This work was funded by the Sheffield Teaching Hospitals Department of Pharmacy and Medicines Management and Small Grants Scheme.

\section{References}

1. American Academy of Sleep Medicine: The International Classification of Sleep Disorders: Diagnostic \& Coding Manual Westchester, IL: American Academy of Sleep Medicine; 2005.

2. Cooper AB, Thornley KS, Young GB, Slutsky AS, Stewart TE, Hanly PJ: Sleep in critically ill patients requiring mechanical ventilation. Chest 2000, 117:809-818.

3. Parthasarathy S, Tobin Martin J: Sleep in the intensive care unit. Intensive Care Med 2004, 30:197-206.

4. Nelson JE, Meier DE, Oei EJ, Nierman DM, Senzel RS, Manfredi PL, Davis SM, Morrison RS: Self-reported symptom experience of critically ill cancer patients receiving intensive care. Crit Care Med 2001, 29:277-282

5. Cajochen C, Kräuchi K, Wirz-Justice A: Role of melatonin in the regulation of human circadian rhythms and sleep. J Neuroendocrinol 2003, 15:432-437.

6. Kuhlwein E, Hauger RL, Irwin MR: Abnormal nocturnal melatonin secretion and disordered sleep in abstinent alcoholics. Biol Psychiatry 2003, 54:1437-1443.

7. Scheer FA, Zeitzer JM, Ayas NT, Brown R, Czeisler CA, Shea SA: Reduced sleep efficiency in cervical spinal cord injury; association with abolished night time melatonin secretion. Spinal Cord 2006, 44:78-81.

8. Shilo L, Dagan Y, Smorjik Y, Weinberg U, Dolev S, Komptel B, Balaum $\mathrm{H}$, Shenkman L: Patients in the intensive care unit suffer from severe lack of sleep associated with loss of normal melatonin secretion pattern. Am J Med Sci 1999, 317:278-281.

9. Frisk U, Olsson J, Nylen P, Hahn RG: Low melatonin excretion during mechanical ventilation in the intensive care unit. Clin Sci 2004, 107:47-53.

10. Olofsson $\mathrm{K}$, Alling $\mathrm{C}$, Lundberg $\mathrm{D}$, Malmros $\mathrm{C}$ : Abolished circadian rhythm of melatonin secretion in sedated and artificially ventilated intensive care patients. Acta Anaesthesiol Scand 2004, 48:679-684.

11. Ibrahim MG, Bellomo R, Hart GK, Norman TR, Goldsmith D, Bates $S$, Egi M: A double-blind placebo-controlled randomised pilot study of nocturnal melatonin in tracheostomised patients. Crit Care Resusc 2006, 8:187-191.

12. Arendt J, Skene DJ: Melatonin as a chronobiotic. Sleep Medicine Rev 2005, 9:25-39.

13. Riker RR, Picard JT, Fraser GL: Prospective evaluation of the Sedation-Agitation Scale for adult critically ill patients. Crit Care Med 1999, 27:1325-1329.

14. Bourne RS, McLaughlin JP, Simpson CC: Benefits on patient's sleep patterns can be studied using a stable oral liquid melatonin formulation. Pharmacy in Practice 2005, 15:246-249.

15. Bourne RS, Mills GH: Sleep disruption in critically ill patients pharmacological considerations. Anaesthesia 2004, 59:374-384.

16. Bourne RS, Mills GH: Melatonin: possible implications for the postoperative and critically ill patient. Intensive Care Med 2006, 32:371-379.

17. Nicholson T, Patel J, Sleigh JW: Sleep patterns in intensive care unit patients: a study using the bispectral index. Crit Care Resusc 2001, 3:86-91.

18. Tung A, Lynch JP, Roizen MF: Use of the BIS monitor to detect onset of naturally occurring sleep. J Clin Monit Comput 2002, 17:37-42.

19. Arendt J, Borbely AA, Franey C, Wright J: The effects of chronic, small doses of melatonin given in the late afternoon on fatigue in man: a preliminary study. Neurosci Lett 1984, 45:317-321.

20. MacFarlane JG, Cleghorn JM, Brown GM, Streiner DL: The effects of exogenous melatonin on the total sleep time and daytime alertness of chronic insomniacs: a preliminary study. Biol Psychiatry 1991, 30:371-376.

21. Bourne RS, Minelli C, Mills GH, Kandler R: Sleep measurement in critical care patients: research and clinical implications. Crit Care 2007, 11:226.

22. Richards KC, Bairnsfather L: A description of night sleep patterns in the critical care unit. Heart Lung 1988, 17:35-42.
23. Richards KC: Effect of a back massage and relaxation intervention on sleep in critically ill patients. Am J Crit Care 1998, 7:288-299.

24. Richards KC, O'Sullivan PS, Phillips RL: Measurement of sleep in critically ill patients. J Nurs Meas 2000, 8:131-144.

25. Ohayon MM, Carskadon MA, Guilleminault C, Vitiello MV: Metaanalysis of quantitative sleep parameters from childhood to old age in healthy individuals: developing normative sleep values across the human lifespan. Sleep 2004, 27:1255-1273.

26. Harrell RG, Othmer E: Postcardiotomy confusion and sleep loss. J Clin Psychiatry 1987, 48:445-446.

27. Bosma K, Ferreyra G, Ambrogio C, Pasero D, Mirabella L, Braghiroli A, Appendini L, Mascia L, Ranieri VM: Patient-ventilator interaction and sleep in mechanically ventilated patients: pressure support versus proportional assist ventilation. Crit Care Med 2007, 35:1048-1054.

28. Harrell FE Jr, Lee KL, Califf RM, Pryor DB, Rosati RA: Regression modelling strategies for improved prognostic prediction. Stat Med 1984, 3:143-152.

29. Hardin KA, Seyal M, Stewart T, Bonekat HW: Sleep in critically ill chemically paralyzed patients requiring mechanical ventilation. Chest 2006, 129:1468-1477.

30. Nieuwenhuijs DJF: Processed EEG in natural sleep. Baillieres Best Pract Res Clin Anaesthesiol 2006, 20:49-56.

31. Shilo L, Dagan Y, Smorjik Y, Weinberg U, Dolev S, Komptel B, Shenkman L: Effect of melatonin on sleep quality of COPD intensive care patients: a pilot study. Chronobiol Int 2000 17:71-76.

32. Fontaine DK: Measurement of nocturnal sleep patterns in trauma patients. Heart Lung 1989, 18:402-410.

33. Aurell J, Elmqvist D: Sleep in the surgical intensive care unit: continuous polygraphic recording of sleep in nine patients receiving postoperative care. $\mathrm{Br}$ Med J 1985, 290:1029-1032.

34. Tonner $P$, Paris A, Scholz J: Monitoring consciousness in intensive care medicine. Baillieres Best Pract Res Clin Anaesthesiol 2006, 20:191-200.

35. Buscemi N, Vandermeer B, Hooton N, Pandya R, Tjosvold L, Hartling L, Vohra S, Klassen TP, Baker G: Efficacy and safety of exogenous melatonin for secondary sleep disorders and sleep disorders accompanying sleep restriction: meta-analysis. Br Med J 2006, 332:385-393.

36. Dollins A, Zhdanova I, Wurtman R, Lynch H, Deng M: Effect of inducing nocturnal serum melatonin concentrations in daytime on sleep, mood, body temperature, and performance. Proc Natl Acad Sci USA 1994, 91:1824-1828.

37. Hartter S, Nordmark A, Rose DM, Bertilsson L, Tybring G, Laine K: Effects of caffeine intake on the pharmacokinetics of melatonin, a probe drug for CYP1A2 activity. $\mathrm{Br} J$ Clin Pharmacol 2003, 56:679-682.

38. DeMuro RL, Nafziger AN, Blask DE, Menhinick AM, Bertino JS Jr: The absolute bioavailability of oral melatonin. J Clin Pharmacol 2000, 40:781-784.

39. Hartter S, Grozinger M, Weigmann H, Roschke J, Hiemke C: Increased bioavailability of oral melatonin after fluvoxamine coadministration. Clin Pharmacol Ther 2000, 67:1-6.

40. Cheng $P$, Morgan E: Hepatic cytochrome $\mathbf{P 4 5 0}$ regulation in disease states. Curr Drug Metab 2001, 2:165-183.

41. Renton K: Cytochrome $\mathbf{P} 450$ regulation and drug biotransformation during inflammation and infection. Curr Drug Metab 2004, 5:235-243.

42. Iguchi $\mathrm{H}$, Kato $\mathrm{Kl}$, lbayashi $\mathrm{H}$ : Melatonin serum levels and metabolic clearance rate in patients with liver cirrhosis. $J$ Clin Endocrinol Metab 1982, 54:1025-1027.

43. Zhdanova IV: Melatonin as a hypnotic: Pro. Sleep Medicine Rev 2005, 9:51-65.

44. Wyatt JK, Dijk DJ, Ritz-de Cecco A, Ronda JM, Czeisler CA: Sleep-facilitating effect of exogenous melatonin in healthy young men and women is circadian-phase dependent. Sleep 2006, 29:609-618.

45. Zhdanova IV, Wurtman RJ, Lynch HJ, Ives JR, Dollins AB, Morabito $C$, Matheson JK, Schomer DL: Sleep-inducing effects of low doses of melatonin ingested in the evening. Clin Pharmacol Ther 1995, 57:552-558.

46. Glass J, Lanctot KL, Herrmann N, Sproule BA, Busto UE: Sedative hypnotics in older people with insomnia: meta-analysis of risks and benefits. BrMed J 2005, 331:1169-1175. 
47. Gabor JY, Cooper AB, Crombach SA, Lee B, Kadikar N, Bettger $\mathrm{HE}$, Hanly PJ: Contribution of the intensive care unit environment to sleep disruption in mechanically ventilated patients and healthy subjects. Am J Respir Crit Care Med 2003, 167:708-715.

48. Pandi-Perumal S, Smits M, Spence W, Srinivasan V, Cardinali D, Lowe A, Kayumov L: Dim light melatonin onset (DLMO): a tool for the analysis of circadian phase in human sleep and chronobiological disorders. Prog Neuropsychopharmacol Biol Psychiatry 2007, 31:1-11.

49. Wallace CJ, Robins J, Alvord LS, Walker JM: The effect of earplugs on sleep measures during exposure to simulated intensive care unit noise. Am J Crit Care 1999, 8:210-219.

50. Arendt J: Melatonin and human rhythms. Chronobiol Int 2006, 23:21-37. 